\title{
Mainline and Water Maser Observations of OH/IR stars in the Arecibo Sky
}

\section{B. M. Lewis}

Arecibo Observatory, Puerto Rico

A complete sample of color selected IRAS sources with $S(25) \geq 2$ Jy in the Arecibo sky and $\left(0^{\circ} \leq \delta \leq 37^{\circ}\right)$ was examined previously for $1612 \mathrm{MHz}$ masers. The resulting set of $\mathrm{OH} / \mathrm{IR}$ stars has now been searched for mainline OH masers at Arecibo (Lewis 1997) and for $22 \mathrm{GHz}$ water masers at Effelsberg (Engels \& Lewis 1996). The high overall detection rate of $61.6 \%$ for the mainlines and $54.8 \%$ for water is partly a result of deployed sensitivity, and partly due to the many blue objects in the sample. But the detectability of both masers improves when $\mathrm{S}(25)>20 \mathrm{Jy}$, as the accompanying tables show. Still the bluest objects exhibit higher (usually $\geq 80 \%$ ) detection rates, while there is a clear decrease in detections from redder shells: this decrease begins circa $(25-12) \mu \mathrm{m}=-0.55$ for mainline masers, and abruptly circa $(25-12) \mu \mathrm{m}=-0.35$ for water. These trends adhere to the descriptive sequence of the chronological scenario for masers in circumstellar shells, though a few proto planetary nebulae and very red OH/IR stars have water masers and a few mainline masers are detected in shells with $(25-12) \mu \mathrm{m} \geq-0.2$.

Flux and Color dependence of Water Detection Rates

$\begin{array}{lllll}(25-12) \mu \mathrm{m} & \mathrm{S}(25)>50 & 50>\mathrm{S}(25)>20 & 20>\mathrm{S}(25)>10 & \mathrm{~S}(25)<10 \\ <-0.40 & 87 \%(20 / 23) & 81 \%(25 / 31) & 69 \%(33 / 48) & 65 \%(79 / 122) \\ >-0.30 & 43 \%(6 / 14) & 33 \%(6 / 18) & 33 \%(9 / 27) & 22 \%(10 / 45) \\ \text { ALL } & 70 \%(31 / 44) & 62 \%(33 / 53) & 51 \%(47 / 92) & 51 \%(99 / 194)\end{array}$

By taking account of water and/or mainline data, we estimate expansion velocities for many OH/IR stars in the sample with just a single $1612 \mathrm{MHz}$ feature. Mainline emission from OH/IR stars is generally weaker than $1612 \mathrm{MHz}$ emission, and it is very rare for all three masers to have their strongest features at exactly the same velocity. Since the extant $\mathrm{OH}$ data from IRAS $05506+2414=\mathrm{BC}$ Tau show a single broad feature at exactly the same velocity from all three lines, this source is probably a pre main sequence object rather than a proto planetary nebula.

IR Flux Dependence of Mainline Detection Rates

$\begin{array}{llll}\mathrm{S}(25) & (25-12) \mu \mathrm{m}<-0.55 & -0.55<(25-12) \mu \mathrm{m}<-0.1 & \text { ALL } \\ 0-5 \mathrm{Jy} & 68 \%(26 / 38) & 26 \%(16 / 61) & 44 \%(46 / 105) \\ 5-10 \mathrm{Jy} & 84 \%(21 / 25) & 47 \%(26 / 55) & 57 \%(51 / 89) \\ \text { 10-20 Jy } & 75 \%(15 / 20) & 57 \%(37 / 65) & 63 \%(58 / 92) \\ >20 \mathrm{Jy} & 82 \%(14 / 17) & 82 \%(53 / 65) & 84 \%(81 / 97)\end{array}$

\section{REFERENCES}

Engels, D. \& Lewis, B. M. 1996, A.\&A. Suppl., 116, 117,

Lewis, B. M., 1997, Ap. J. Suppl., April 